\title{
How to Predict Congruential Generators
}

\author{
Hugo Krawczyk \\ Computer Science Dept. \\ Technion \\ Haifa, Israel
}

\begin{abstract}
In this paper we show how to predict a large class of pseudorandom number generators. We consider congruential generators which output a sequence of integers $s_{0}, s_{1}, \ldots$ where $s_{i}$ is computed by the recurrence $s_{i} \equiv \sum_{j=1}^{k} \alpha_{j} \Phi_{j}\left(s_{0}, s_{1}, \ldots, s_{i-1}\right)(\bmod m)$ for integers $m$ and $\alpha_{j}$, and integer functions $\Phi_{j}, j=1, \ldots, k$. Our predictors are efficient, provided that the functions $\Phi_{j}$ are computable (over the integers) in polynomial time. These predictors have access to the elements of the sequence prior to the element being predicted, but they do not know the modulus $m$ or the coefficients $\alpha_{j}$ the generator actually works with. This extends previous results about the predictability of such generators. In particular, we prove that multivariate polynomial generators, i.e. generators where $s_{i} \equiv P\left(s_{i-n}, \ldots, s_{i-1}\right)(\bmod m)$, for a polynomial $P$ of fixed degree in $n$ variables, are efficiently predictable.
\end{abstract}

\section{INTRODUCTION}

A number generator is a deterministic algorithm that given a sequence of initial values, outputs an (infinite) sequence of numbers. Some generators, called pseudorandom number generators are intended to output sequences of numbers having some properties encountered in truly random sequences. Such generators appear in diverse applications as Probabilistic Algorithms, Monte Carlo Simulations, Cryptography, etc. For cryptographic applications a crucial property for the sequences generated is their unpredictability. That is, the next element generated should not be efficiently predictable, even given the entire past sequence. Efficiency is measured both by the number of prediction mistakes and the time taken to compute each prediction. (A formal definition of an efficient predictor is given in section 2). 
A pseudorandom number generator that has received much attention is the so called linear congruential generator, an algorithm that on input integers $a, b, m, s_{0}$ outputs a sequence $s_{1}, s_{2}, \cdots$ where

$$
s_{i} \equiv a s_{i-1}+b(\bmod m) .
$$

Knuth [13] extensively studied some statistical properties of these generators.

Boyar [16] proved that linear congruential generators are efficiently predictable even when the coefficients and the modulus are unknown to the predictor. Later, Boyar [3] extended her method, proving the predictability of a large family of generators. She considered general congruential generators where the element $s_{i}$ is computed as

$$
s_{i} \equiv \sum_{j=1}^{k} \alpha_{j} \Phi_{j}\left(s_{0}, s_{1}, \cdots, s_{i-1}\right)(\bmod m)
$$

for integers $m$ and $\alpha_{j}$, and computable integer functions $\Phi_{j}, j=1, \ldots, k$. She showed that these sequences can be predicted, for some class of functions $\Phi_{j}$, by a predictor knowing these functions and able to compute them, but not given the coefficients $\alpha_{j}$ or the modulus $m$. Boyar's method requires that the functions $\Phi_{j}$ have the unique extrapolation property. The functions $\Phi_{1}, \Phi_{2}, \cdots, \Phi_{k}$ have the unique extrapolation property with length $r$, if for every pair of generators working with the above set of functions, the same modulus $m$ and the same initial values, if both generators coincide in the first $r$ values generated, then they output the same infinite sequence. Note that these generators need not be identical (i.e. they may have different coefficients).

The number of mistakes made by Boyar's predictors depends on the extrapolation length. Therefore, her method yields efficient predictors provided that the functions $\Phi_{j}$ have a small extrapolation length. The linear congruential generator is an example of a generator having the extrapolation property (with length 2). Boyar proved this property also for two extensions of the linear congruential generator. Namely, the generators in which the element $s_{i}$ satisfies the recurrence

and those for which

$$
s_{i} \equiv \alpha_{1} s_{i-k}+\cdots+\alpha_{k} s_{i-1}(\bmod m)
$$

$$
s_{i} \equiv \alpha_{1} s_{i-1}^{2}+\alpha_{2} s_{i-1}+\alpha_{3}(\bmod m)
$$

The first case with length $k+1$, the second with length 3 . She also conjectured the predictability of generators having a polynomial recurrence:

$$
s_{i} \equiv p\left(s_{i-1}\right)(\bmod m)
$$

for an unknown polynomial $p$ of fixed (and known) degree.

A natural generalization of the above examples is a generator having a multivariate polynomial recurrence, that is a generator outputting a sequence $s_{0}, s_{1}, \ldots$ where

$$
s_{i} \equiv P\left(s_{i-n}, \ldots, s_{i-1}\right)(\bmod m)
$$

for a polynomial $P$ in $n$ variables. Note that for polynomials $P$ of fixed degree and fixed $n$, the recurrence is a special case of the general congruential generators. Lagarias and Reeds [15] showed that multivariate polynomial recurrences have the unique 
extrapolation property. Furthermore, for the case of a one-variable polynomial of degree $d$, they proved this property with length $d+1$, thus settling Boyar's conjecture concerning the efficient predictability of such generators. However, for the general case they did not give a bound on the length for which these recurrences are extrapolatable (neither a way to compute this length). Thus, unfortunately, Boyar's method does not seem to yield an efficient predicting algorithm for general multivariate polynomial recurrences (since it is not guaranteed to make a small number of mistakes but only a finite number of them, depending on the length of the extrapolation).

In this paper we show how to predict any general congruential generator, i.e. any generator of the form (1). The only restriction on the functions $\Phi_{j}$ is that they are computable in polynomial time when working over the integers. This condition is necessary to guarantee the efficiency of our method. (The same is required in Boyar's method). Thus, we remove the necessity of the unique extrapolation property, and extend the predictability results to a very large class of generators. In particular, we show that multivariate polynomial recurrence generators are efficiently predictable.

Our predicting technique is based on ideas from Boyar's method, but our approach to the prediction problem is somewhat different. Boyar's method tries to simulate the generator by "discovering" its secrets: the modulus $m$ and the coefficients $\alpha_{j}$ that the generator works with. Instead, our algorithm uses only the knowledge that these coefficients exist, but does not try to find them. Some algebraic techniques introduced by Boyar when computing over the integers, are extended by us to work also when computing over the ring of integers modulo $m$.

\section{DEFINITIONS AND NOTATION}

Definition: A number generator is an algorithm that given $n_{0}$ integer numbers, called the initial values and denoted $s_{-n_{\mathrm{a}}}, \cdots, s_{-1}$, outputs an infinite sequence of integers $s_{0}, s_{1}, \ldots$ where each element $s_{i}$ is computed deterministicly from the previous elements, including the initial values.

For example, a generator of the form $s_{i} \equiv \alpha_{1} s_{i-k}+\cdots+\alpha_{k} s_{i-1}(\bmod m)$ requires a set of $k$ initial values to begin computing the first elements $s_{0}, s_{1}, \cdots$ of the sequence. Thus, for this example $n_{0}=k$.

Definition: A (general) congruential generator is a number generator for which the $i$-th element of the sequence is a $\{0, \ldots, m-1\}$-valued number computed by the congruence

$$
s_{i} \equiv \sum_{j=1}^{k} \alpha_{j} \Phi_{j}\left(s_{-n_{0}}, \cdots, s_{-1}, s_{0}, \cdots, s_{i-1}\right)(\bmod m)
$$

where $\alpha_{j}$ and $m$ are arbitrary integers and $\Phi_{j}, 1 \leq j \leq k$, is a computable integer function. For a given set of $k$ functions $\Phi=\left\{\Phi_{1}, \Phi_{2}, \ldots, \Phi_{k}\right\}$ a congruential generator working with these functions (and arbitrary coefficients and modulus) will be called a $\Phi$-generator. 
Example: Consider a generator which outputs a sequence defined by a multivariate polynomial recurrence, i.e. $s_{i} \equiv P\left(s_{i-n}, \ldots, s_{i-1}\right)(\bmod m)$, where $P$ is a polynomial in $n$ variables and fixed degree $d$. Such a generator is a $\Phi$-generator in which each function $\Phi_{j}$ represents a monomial in $P$ and $\alpha_{j}$ are the corresponding coefficients. In this case we have $k=\left(\begin{array}{c}n+d \\ d\end{array}\right)$, and the functions (monomials) $\Phi_{j}$ are applied to the last $n$ elements in the sequence.

Note that in the above general definition, the functions $\Phi_{j}$ work on sequences of elements, so the number of arguments for these functions may be variable. Some matrix notation will be more convenient.

Notation: $s(i)$ will denote the vector of elements (including the initial values) until the element $s_{i}$, i.e.

$$
s(i)=\left(s_{-n_{0},} \cdots, s_{-1}, s_{0}, \cdots, s_{i}\right) \quad i=-1,0,1,2 \cdots
$$

Thus, $\Phi_{j}\left(s_{-n_{0}}, \cdots, s_{-1}, s_{0}, \cdots, s_{i-1}\right)$ will be written as $\Phi_{j}(s(i-1))$.

Let $\alpha$ denote the vector $\left(\alpha_{1}, \alpha_{2}, \cdots, \alpha_{k}\right)$ and $B_{i}, i \geq 0$, denote the column vector

$$
B_{i}=\left[\begin{array}{c}
\Phi_{1}(s(i-1)) \\
\Phi_{2}(s(i-1)) \\
: \\
\Phi_{k}(s(i-1))
\end{array}\right]
$$

Then we can rewrite the $\Phi$-generator's recurrence as

$$
s_{i} \equiv \alpha \cdot B_{i}(\bmod m)
$$

Here, and in the sequel, $\cdot$ denotes matrix multiplication.

Finally, $B(i)$ will denote the matrix

$$
B(i)=\left[B_{0} B_{1} \cdots B_{i}\right]
$$

For complexity considerations we refer to the size of the prediction problem as given by the size of the modulus $m$ and the number $k$ of coefficients the generator actually works with. (Note that the coefficients as well as the elements output by the generator have size at most $\log m$ ). We consider as efficient generators for which the functions $\Phi_{j}, 1 \leq j \leq k$, are computable in time polynomial in $\log m$ and $k$. Also the efficiency of a predictor will be measured in terms of these parameters, which can be seen as measuring the amount of information hidden from the predictor.

We shall be concerned with the complexity of the functions $\Phi_{j}$ when acting on the vectors $s(i)$, but computed over the integers (and not reduced modulo $m$ ). This will be referred to as the non-reduced complexity of the functions $\Phi_{j}$. The performance of our predicting algorithm will depend on this complexity. 
Definition: $\Phi$-generators having non-reduced time-complexity polynomial in $\log m$ and $k$ are called non-reduced polynomial-time $\Phi$-generators.

Next we define the basic concept, throughout this paper, of a predictor:

Definition: A predictor for a $\Phi$-generator is an algorithm that interacts with the $\Phi$ generator in the following way. The predictor gets as input the initial values that the generator is working with. For $i=0,1,2, \ldots$ the predictor outputs its prediction for the element $s_{i}$ and the generator responds with the true value of $s_{i}$.

An efficient predictor (for a $\Phi$-generator) is a predictor for which there exist polynomials $P$ and $Q$ such that

1) the computation time for every prediction is bounded by $P(k, \log m)$

2 ) the number of prediction mistakes is bounded by $Q(k, \log m)$

Observe that when computing its prediction for $s_{i}$ the predictor has seen the entire segment of the sequence before $s_{i}$, and the initial values. The only secret information kept by the generator is the coefficients and the modulus. If the generator is not given the initial values then our method cannot be applied to arbitrary $\Phi$-generators. However, in typical cases (including the multivariate polynomial recurrence) generators have recurrences depending only on the last $n_{0}$ elements, for some constant $n_{0}$. In this case the predictor may consider the first $n_{0}$ elements generated as initial values, and begin predicting after the generator outputs them.

\section{THE PREDICTING ALGORITHM}

The predictor tries to infer the element $s_{i}$ from knowledge of all the previous elements of the sequence, including the initial values. It does not know the modulus $m$ the generator is working with, so it uses different estimates for this $m$. Its first estimate is $\hat{m}=\infty$, i.e. the predictor begins by computing over the integers. After some portion of the sequence is revealed, and taking advantage of possible prediction mistakes, a new (finite) estimate $\hat{m}_{0}$ for $m$ is computed. Later on, new values for $\hat{m}$ are computed in such a way that each $\hat{m}$ is a (non-trivial) divisor of the former estimate, and all are multiples of the actual $m$. Eventually $\hat{m}$ may reach the true value of $m$. (For degenerate cases, like a generator producing a constant sequence, it may happen that $m$ will never be reached but this will not effect the prediction capabilities of the algorithm).

We shall divide the predicting algorithm into two stages. The first stage is when working over the integers, i.e. $\hat{m}=\infty$. The second one is after the first finite estimate $m_{0}$ was computed. The distinction between these two stages is not essential, but some technical reasons make it convenient. In fact, the algorithm is very similar for both stages. 
The idea behind the algorithm is to find linear dependencies among the columns of the matrix $B(i)$ and to use these dependencies in making the prediction of the next element $s_{i}$. More specificly, we try to find a representation of $B_{i}$ as a linear combination (modulo the current $\hat{m}$ ) of the previous $B_{j}$ 's (that are known to the predictor at this time). If such a combination exists, we apply it to the previous elements in the sequence (i.e. previous $s_{j}$ 's) to obtain our prediction for $s_{i}$. If not correct, we made a mistake but gain information that allows us to refine the modulus $m$. A combination as above will not exist if $B_{i}$ is independent of the previous columns. We show that under a suitable definition of independence, the number of possible independent $B_{i}$ 's cannot be too large. Therefore only a small number of mistakes is possible, allowing us to prove the efficiency of the predictor.

The number of mistakes made by the predictor, until it is able to refine the current $\hat{m}$, will be bounded by a polynomial in the size of this $\hat{m}$. Also the total number of distinct moduli $\hat{m}$ computed during the algorithm is bounded by the size of the first (finite) $m_{0}$. Thus, the total number of possible mistakes is polynomial in this size, which in turn is determined by the length of the output of the non-reduced functions $\Phi_{j}$. This is the reason for which the non-reduced complexity of these functions is required to be polynomial in the size of the true $m$ and $k$. In this case the total number of mistakes made by the predictor will also be polynomial in these parameters. The same is true for the computation time of every prediction.

The algorithm presented here is closely related to Boyar's [3]. Our first stage is exactly the same as the first stage there. That is, the two algorithms begin by computing a multiple of the modulus $m$. Once this is accomplished, Boyar's strategy is to find a set of coefficients $\left\{\alpha_{j}^{\prime}\right\}_{j=1}^{k}$ and a sequence of moduli $m^{h}$ which are refined during the algorithm until no more mistakes are made. For proving the correctness and efficiency of her predictor, it is required that the generator satisfies the unique extrapolation property (mentioned in the Introduction). In our work, we do not try to find the coefficients. Instead, we extend the ideas of the first stage, and apply them also in the second stage. In this way the need for an extrapolation property is avoided, allowing the extensions of the predictability results.

\subsection{First Stage}

Let us describe how the predictor computes its prediction for $s_{i}$. At this point the predictor knows the whole sequence before $s_{i}$, i.e. $s(i-1)$, and so far it has failed to compute a finite multiple of the modulus $m$, so it is still working over the integers. In fact, the predictor is able at this point to compute all the vectors $B_{0}, B_{1}, \cdots, B_{i}$, since they depend only on $s(i-1)$. Moreover, our predictor keeps at this point, a submatrix of $B(i-1)$, denoted by $\overline{B(i-1)}$, of linearly independent (over the rationals) columns. (For every $i$, when predicting the element $s_{i}$, the predictor checks if the column $B_{i}$ is independent of the previous ones. If this is the case then $B_{i}$ is added to $\overline{B(i-1)}$ to form 
$\overline{B(i)})$. Finally, let us denote by $\overline{s(i-1)}$ the corresponding subvector of $s(i-1)$, having the entries indexed with the same indices appearing in $\overline{B(i-1)}$.

\section{Prediction of $s_{i}$ in the first stage:}

The predictor begins by computing the (column) vector $B_{i}$. Then, it solves, over the rationals, the system of equations

$$
\overline{B(i-1)} \cdot x=B_{i}
$$

If no solution exists, $B_{i}$ is independent of the columns in $\overline{B(i-1)}$ so it sets

$$
\overline{B(i)}=\left[\overline{B(i-1)} B_{i}\right]
$$

and it fails to predict $s_{i}$.

If a solution exists, let $c$ denote the solution (vector) computed by the predictor. The prediction for $s_{i}$, denoted $s_{i}$, will be

$$
\hat{s_{i}}=\overline{s(i-1)} \cdot c
$$

The predictor, once having received the true value for $s_{i}$, checks whether this prediction is correct or not (observe that the prediction $s_{i}$ as computed above may not even be an integer). If correct, it has succeeded and goes on predicting $s_{i+1}$. If not, i.e. $s_{i} \neq s_{i}$, the predictor has made a mistake, but now it is able to compute $\hat{m}_{0} \neq \infty$, the first multiple of the modulus $m$, as follows. Let $l$ be the number of columns in matrix $\overline{B(i-1)}$ and let the solution $c$ be

$$
c=\left[\begin{array}{c}
c_{1} / d_{1} \\
c_{2} / d_{2} \\
: \\
c_{l} / d_{l}
\end{array}\right]
$$

Now, let $d$ denote the least common multiple of the dominators in these fractions, i.e. $d=l c m\left(d_{1}, \cdots, d_{l}\right)$. The value of $\hat{m}_{0}$ is computed as follows

$$
\hat{m_{0}}=\left|d s_{i}-d s_{i}\right| \text {. }
$$

Observe that $\hat{m}_{0}$ is an integer, even if $\hat{s}_{i}$ is not. Moreover this integer is a multiple of the true modulus $m$ the generator is working with (see Lemma 1 below).

Once $m_{0}$ is computed, the predictor can begin working modulo this $\hat{m}_{0}$. So the first stage of the algorithm is terminated and it goes on into the second one.

The main facts concerning the performance of the predicting algorithm during the first stage are summarized in the next Lemma. 


\section{Lemma 1:}

a) The number $m_{0}$ computed at the end of the first stage is a nonzero multiple of the modulus $\boldsymbol{m}$.

b) The number of mistakes made by the predictor in the first stage is at most $k+1$.

c) For non-reduced polynomial time $\Phi$-generators, the prediction time for each $s_{i}$ during the first stage is polynomial in $\log m$ and $k$.

d) For non-reduced polynomial time $\Phi$-generators, the size of $m_{0}$ is polynomial in log $m$ and $k$. More precisely, let $M$ be an upper bound on the output of each of the functions $\Phi_{j}, j=1, \ldots, k$, working on $\{0, \ldots, m-1\}$-valued integers. Then, $m_{0} \leq(k+1) k^{k / 2} m M^{k}$.

\section{Proof:}

a) From the definition of the generator we have the congruence $s_{j} \equiv \alpha \cdot B_{j}(\bmod m$ ) for all $j \geq 0$, therefore

$$
\overline{s(i-1)} \equiv \alpha \cdot \overline{B(i-1)}(\bmod m)
$$

Thus,

$$
\begin{aligned}
d s_{i} & =d \overline{s(i-1)} \cdot c & & \left(\text { by definition of } s_{i}\right) \\
& \equiv d \alpha \cdot \overline{B(i-1)} \cdot c(\bmod m) & & (\text { by }(3)) \\
& =d \alpha \cdot B_{i} & & \left(c \text { is a solution to } \overline{B(i-1)} \cdot x=B_{i}\right) \\
& =d s_{i}(\bmod m) & & \text { (By definition of } \left.s_{i}(2)\right)
\end{aligned}
$$

So we have shown that $d \hat{s}_{i} \equiv d s_{i}(\bmod m)$. Observe that it cannot be the case that $d s_{i}=d s_{i}$, because this implies $s_{i}=s_{i}$, contradicting the incorrectness of the prediction. Thus, we have proved that $\hat{m}_{0}=\left|d s_{i}-d s_{i}\right|$ is indeed a nonzero multiple of $m$.

b) The possible mistakes in the first stage are when a rational solution to the system of equations $\overline{B(i-1)} \cdot x=B_{i}$ does not exist, or when such a solution exists but our prediction is incorrect. The last case will happen only once because after that occurs the predictor goes into the second stage. The first case cannot occur "too much". Observe that the matrices $B(j)$ have $k$ rows, thus the maximal number of independent columns (over the rationals) is at most $k$. So the maximal number of mistakes made by the predictor in the first stage is $k+1$.

c) The computation time for the prediction of $s_{i}$ is essentially given by the time spent computing $B_{i}$ and solving the above equations. The functions $\Phi_{j}$ are computable in time polynomial in $\log m$ and $k$, so the computation of the vector $B_{i}$ is also polynomial in $\log m$ and $k$. The complexity of solving the system of equations, over the rationals, is polynomial in $k$ and in the size of the entries of $\overline{B(i-1)}$ and $B_{i}$ (see [8], [18, Ch.3]). These entries are determined by the output of the (non-reduced) functions $\Phi_{j}$, and therefore their size is bounded by a polynomial in $\log m$ and $k$. Thus, the total complexity of 
the prediction step is polynomial in $\log m$ and $k$, as required.

d) As pointed out in the proof of claim $c$ ), a solution to the system of equations in the algorithm, can be found in time bounded polynomially in $\log m$ and $k$. In particular this guarantees that the size of the solution will be polynomial in $\log m$ and $k$. (By size we mean the size of the denominators and numerators in the entries of the solution vector.) Clearly, by the definition of $m_{0}$, the polynomiality of the size of the solution $c$ implies that the size of $m_{0}$ is itself polynomial in $\log m$ and $k$.

The explicit bound on $\hat{m}_{0}$ can be derived as follows. Using Cramer's rule we get that the solution $c$ to the system $\overline{B(i-1)} \cdot x=B_{i}$, can be represented as $c=\left(c_{1} / d, \ldots, c_{l} / d\right)$ where each $c_{j}$ and $d$ are determinants of $l$ by $l$ submatrices in the above system of equations. Let $D$ be the maximal possible value of a determinant of such a matrix. We have that $d s_{i}=d \overline{s(i-1)} c \leq l m D$ (here $m$ is a bound on $\overline{s(i-1)}$ entries) and $d s_{i} \leq m D$, then $m_{0}=\left|d s_{i}-d s_{i}\right| \leq(l+1) m D$. In order to bound $D$ we use Haddamard's inequality which states that each $n$ by $n$ matrix $A=\left(a_{i j}\right)$ satisfies $\operatorname{det}(A) \leq \prod_{i=1}^{n}\left(\sum_{j=1}^{n} a_{i j}^{2}\right)^{1 / 2}$. In our case the matrices are of order $l$ by $l$, and the entries to the system are bounded by $M$ (the bound on $\Phi_{j}$ output). Thus, $D \leq \prod_{i=1}^{l}\left(\sum_{j=1}^{l} M^{2}\right)^{1 / 2}=\left(l M^{2}\right)^{i / 2}$, and we get

$$
m_{0} \leq(l+1) m D \leq(l+1) m\left(l M^{2}\right)^{l / 2} \leq(k+1) k^{k / 2} m M^{k}
$$

The last inequality follows since $l \leq k$.

\subsection{Second Stage}

After having computed $\hat{m}_{0}$, the first multiple of $m$, we proceed to predict the next elements of the sequence, but now working modulo a finite $m$. The prediction step is very similar to the one described for the first stage. The differences are those that arise from the fact that the computations are modulo an integer. In particular the equations to be solved will not be over a field (in the first stage it was over the rationals), but rather over the ring of residues modulo $\hat{m}$. Let us denote the ring of residues modulo $n$ by $Z_{n}$. In the following definition we extend the concept of linear dependence to these rings.

Definition: Let $v_{1}, v_{2}, \ldots, v_{l}$ be a sequence of $l$ vectors with $k$ entries from $Z_{n}$. We say that this sequence is weakly linearly dependent $\bmod n$ if $v_{1}=0$ or there exists an index $i, 2 \leq i \leq l$, and elements $c_{1}, c_{2}, \ldots, c_{i-1} \in Z_{n}$, such that $v_{i} \equiv c_{1} v_{1}+c_{2} v_{2}+\cdots+c_{i-1} v_{i-1}(\bmod n)$. Otherwise, we say that the sequence is weakly linearly independent.

Note that the order here is important. Unlike the case in the traditional definition over a field, in the above definition it is not equivalent to say that some vector in the set can be written as a linear combination of the others. Another important difference is that it is not true in general, that $k+1$ vectors of $k$ components over $Z_{n}$ must contain a 
dependent vector. Fortunately, a slightly weaker statement does hold.

Theorem 2: Let $v_{1}, v_{2}, \ldots, v_{l}$ be a sequence of $k$-dimensional vectors over $Z_{n}$. If the sequence is weakly linearly independent $\bmod n$, then $l \leq k \log _{q} n$, where $q$ is the smallest prime dividing $n$.

Proof: Let $v_{1}, v_{2}, \ldots, v_{l}$ be a sequence of $l$ vectors from $Z_{n}^{k}$, and suppose this sequence is weakly linearly independent $\bmod n$. Consider the set

$$
V=\left\{\sum_{i=1}^{l} c_{i} v_{i}(\bmod n): c_{i} \in\{0,1, \cdots, q-1\}\right\}
$$

We shall show that this set contains $q^{l}$ different vectors. Equivalently, we show that no two (different) combinations in $V$ yield the same vector.

Claim: For every $c_{i}, c_{i}^{\prime} \in\{0,1, \cdots, q-1\}, 1 \leq i \leq l$, if $\sum_{i=1}^{l} c_{i} v_{i} \equiv \sum_{i=1}^{l} c_{i}^{\prime} v_{i}(\bmod m)$ then $c_{i}=c_{i}^{\prime}$ for $i=1,2, \ldots, l$.

Suppose this is not true. Then we have $\sum_{i=1}^{l}\left(c_{i}-c_{i}^{\prime}\right) v_{i} \equiv 0(\bmod n)$. Denote $c_{i}-c_{i}^{\prime}$ by $d_{i}$. Let $t$ be the maximal index for which $d_{t} \neq 0$. This number $d_{t}$ satisfies $-q<d_{t}<q$, so it has an inverse modulo $n$ (recall that $q$ is the least prime divisor of $n$ ), denoted $d_{l}^{-1}$. It follows that $v_{t}=\sum_{i=1}^{i-1}-d_{t}^{-1} d_{i} v_{i}(\bmod n)$ contradicting the independence of $v_{t}$, and thus proving the claim.

Hence, $|V|=q^{l}$ and therefore

$$
q^{l}=|V| \leq\left|Z_{n}^{k}\right|=n^{k}
$$

which implies $l \leq k \log _{q} n$, proving the Theorem.

With the above definition of independence in mind, we can define the matrix $\overline{B(i)}$ as a submatrix of $B(i)$, in which the (sequence of) columns are weakly linearly independent $\bmod m$. Note that $\hat{m}$ will have distinct values during the algorithm, so when writing $\overline{B(i)}$ we shall refer to its value modulo the current $m$.

\section{Prediction of $s_{i}$ in the second stage:}

Let us describe the prediction step for $s_{i}$ when working modulo $m$. In fact, all we need is to point out the differences with the process described for the first stage.

As before, we begin by computing the vector $B_{i}$ (now reduced modulo $m$ ), and solving the system of equations

$$
\overline{B(i-1)} \cdot x \equiv B_{i}(\bmod \hat{m})
$$

We stress that this time we are looking for a solution over $Z_{m}$. In case a solution does not exist, we fail to predict, exactly as in the previous case. As before, the vector $B_{i}(\bmod m)$ 
is added to $\overline{B(i-1)}$ to form the matrix $\overline{B(i)}$. If a solution does exist, we output our prediction, computed as before, but the result is reduced mod $\hat{m}$. Namely, we set $s_{i}=\overline{s(i-1)} \cdot c(\bmod m)$, where $c$ is a solution to the above system of modular equations. If the prediction is correct, we proceed to predict the next element $s_{i+1}$. If not, we take advantage of this error to update $m$. This is done by computing

$$
m^{\prime}=\operatorname{gcd}\left(\hat{m}, \hat{s_{i}}-s_{i}\right)
$$

This $m^{\prime}$ will be the new $\hat{m}$ we shall work with in the coming predictions.

To see that the prediction algorithm as described here, is indeed an efficient predictor, we have to prove the following facts summarized in Lemma 3. (Lemma 3 is analogous to Lemma 1 for the second stage).

Lemma 3: The following claims hold for a predictor predicting a non-reduced polynomial time $\Phi$-generator.

a) The number $m$ computed above is a nontrivial divisor of $m$ and a multiple of the modulus $m$.

b) Let $m_{0}$ be the modulus computed at the end of the first stage. The total number of mistakes made by the predictor during the second stage is bounded by $(k+1) \log m_{0}$, and then polynomial in $\log m$ and $k$.

c) The prediction time for each $s_{i}$ during the second stage is polynomial in $\log m$ and $k$.

\section{Proof:}

a) Recall that $m^{\prime}=g c d\left(m^{\prime}, s_{i}-s_{i}\right)$, so it is a divisor of $\hat{m}$. It is a nontrivial divisor because $s_{i}$ and $s_{i}$ are reduced $\bmod \hat{m}$ and $m$ respectively, and then their difference is strictly less than $\hat{m}$. It cannot be zero because $\hat{s}_{i} \neq s_{i}$, as follows from the incorrectness of the prediction. The proof that $m^{\prime}$ is a multiple of $m$ is similar to that of claim a) of Lemma 1 . It is sufficient to show that $\hat{s}_{i}-s_{i}$ is a multiple of $m$, since $m$ is itself a multiple of $m$. We show this by proving $\hat{s}_{i} \equiv s_{i}(\bmod m)$ :

$$
\begin{aligned}
s_{i} & \equiv \overline{s(i-1)} \cdot c(\bmod m) & & \left(\text { by definition of } s_{i}\right) \\
& \equiv \alpha \cdot \overline{B(i-1)} \cdot c(\bmod m) & & (\text { by }(3)) \\
& \equiv \alpha \cdot B_{i}(\bmod m) & & \left(c \text { is a solution to } \overline{B(i-1)} \cdot x \equiv B_{i}(\bmod m)\right) \\
& \equiv s_{i}(\bmod m) & & \text { (By definition of } \left.s_{i}(2)\right)
\end{aligned}
$$

As $m$ divides $m$, claim a) follows.

b) The possible mistakes during the second stage are of two types. Mistakes of the first type happen when a solution to the above congruential equations does not exist. This implies the independence modulo the current $m$ of the corresponding $B_{i}$. In fact, this $B_{i}$ is also independent mod $m_{0}$. This follows from the property that every $m$ is a divisor of $m_{0}$. By Theorem 2, we have that the number of weakly linearly independent vectors mod $m_{0}$ is at most $k \log m_{0}$. Therefore the number of mistakes by lack of a solution is bounded by 
this quantity too. The second type of mistake is when a solution exists but the computed prediction is incorrect. Such a mistake can occur only once per $m$. After it occurs, a new $m$ is computed. Thus, the total number of such mistakes is as the number of different $m$ 's computed during the algorithm. These $m$ 's form a decreasing sequence of positive integers in which every element is a divisor of the previous one. The first (i.e. largest) element is $m_{0}$ and then the length of this sequence is at most $\log m_{0}$. Consequently, the total number of mistakes during the second stage is at most $(k+1) \log \hat{m}_{0}$, and by Lemma 1 claim d) this number is polynomial in $\log m$ and $k$.

c) By our assumption of the polynomiality of the functions $\Phi_{j}$ when working on the vectors $s(i)$, it is clear that the computation of each $B_{i}(\bmod \hat{m})$, takes time that is polynomial in $\log m$ and $k$. We only need to show that a solution to $\overline{B(i-1)} \cdot x \equiv B_{i}(\bmod \hat{m})$ can be computed in time polynomial in $\log m$ and $k$. A simple method for the solution of a system of linear congruences like the above, is described in [6] (and [3]). This method is based on the computation of the Smith Normal Form of the coefficients matrix in the system. This special matrix and the related transformation matrices, can be computed in polynomial time, using an algorithm of [12]. Thus, finding a solution to the above system (or deciding that none exists) can be accomplished in time polynomial in $\log m$ and $k$. Therefore the whole prediction step is polynomial in these parameters.

Combining Lemmas 1 and 3 we get

Theorem 4: For every non-reduced polynomial-time $\Phi$-generator the predicting algorithm described above is an efficient predictor. The number of prediction mistakes is at most $(k+1)\left(\log m_{0}+1\right)=O\left(k^{2} \log (k m M)\right)$, where $\hat{m}_{0}$ is the first finite modulus computed by the algorithm, and $M$ is an upper bound on the output of each of the functions $\Phi_{j}, j=1, \ldots, k$, working over integers in the set $\{0, \ldots, m-1\}$.

\section{As a special case we get}

Corollary: Every multivariate polynomial recurrence generator is efficiently predictable. The number of prediction mistakes for a polynomial recurrence in $n$ variables and degree $d$ is bounded by $O\left(k^{2} \log \left(k m^{d}\right)\right)$, where $k=\left(\begin{array}{c}n+d \\ d\end{array}\right)$.

Proof: A multivariate polynomial recurrence is a special case of a $\Phi$-generator with $M<m^{d}$, as each monomial is of degree at most $d$ and it is computed on integers less than $m$. Therefore, by Lemma 1 d) we get $\hat{m}_{0}<(k+1) k^{k / 2} m^{d k+1}$. The number $k$ of coefficients is as the number of possible monomials in such a polynomial recurrence which is $\left(\begin{array}{c}n+d \\ d\end{array}\right)$. The bound on the number of mistakes follows by substituting these parameters in the general bound of Theorem 4 . 
Remark: Notice that the number $k$ of coefficients equals the number of possible monomials in the polynomial. For general polynomials in $n$ variables and of degree $d$, this number is $\left(\begin{array}{c}n+d \\ d\end{array}\right)$. Nevertheless, if we consider special recurrences in which not every monomial is possible, e.g. $s_{i} \equiv \alpha_{1} s_{i-n}^{2}+\cdots+\alpha_{n} s_{i-1}^{2}(\bmod m)$, then the number $k$ may be much smaller, and then a better bound on the number of mistakes for such cases is derived.

\section{VECTOR-VALUED RECURRENCES}

The most interesting subclass of $\Phi$-generators is the class of multivariate polynomial recurrence generators mentioned in previous sections. Lagarias and Reeds [15] studied a more general case of polynomial recurrences in which a sequence of $n$ dimensional vectors over $Z_{m}$ is generated, rather than a sequence of $Z_{m}$ elements as in our case. These vector-valued polynomial recurrences have the form

$$
\bar{s}_{i} \equiv\left(P_{1}\left(\bar{s}_{i-1,1}, \ldots, \bar{s}_{i-1, n}\right)(\bmod m), \ldots, P_{n}\left(\bar{s}_{i-1,1}, \ldots, \bar{s}_{i-1, n}\right)(\bmod m)\right)
$$

where each $P_{l}, 1 \leq l \leq n$, is a polynomial in $n$ variables and of maximal degree $d$. Clearly, these recurrences extend the single-valued case, since for any multivariate polynomial $P$ which generates a sequence $\left(s_{i}\right\}_{i=\infty}^{\infty}$ of $Z_{m}$ elements, one can consider the sequence of vectors $\bar{s}_{i}=\left(s_{i}, s_{i-1}, \ldots, s_{i-n+1}\right)$ where $\bar{s}_{i}=\left(P\left(s_{i-1}, \ldots, s_{i-n}\right)(\bmod m), s_{i-1}, \ldots, s_{i-n+1}\right)$.

The vector-valued polynomial recurrences can be generalized in terms of $\Phi$ generators as follows. Consider $n$ congruential generators $\Phi^{(1)}, \ldots, \Phi^{(n)}$, where $\Phi^{(l)}=\left\{\Phi_{j}^{(l)}\right\}_{j=1}^{k}$, and for each $j, l, \Phi_{j}^{(l)}$ is a function in $n$ variables. For any set $\left\{\alpha_{j}^{(l)}: 1 \leq j \leq k, 1 \leq l \leq n\right\}$ of coefficients and modulus $m$, we define a vector-valued generator which outputs a sequence of vectors $\bar{s}_{0}, \bar{s}_{1}, \ldots$, where each $\overline{s_{i}}=\left(\bar{s}_{i, 1}, \ldots, \bar{s}_{i, n}\right) \in Z_{m}^{n}$ is generated by the recurrence

$\bar{s}_{i} \equiv\left(\sum_{j=1}^{k} \alpha_{j}^{(1)} \Phi_{j}^{(1)}\left(\bar{s}_{i-1,1}, \ldots, \bar{s}_{i-1, n}\right)(\bmod m), \ldots, \sum_{j=1}^{k} \alpha_{j}^{(n)} \Phi_{j}^{(n)}\left(\bar{s}_{i-1,1}, \ldots, \bar{s}_{i-1, n}\right)(\bmod m)\right)$

It is easy to see that vector-valued recurrences of the form (4) can be predicted in a similar way to the single-valued recurrences studied in the previous section. One can apply the prediction method of Section 3 to each of the "sub-generators" $\Phi_{j}^{(l)}, l=1, \ldots, n$. Notice that $\bar{s}_{i}$ is computed by applying the functions $\Phi_{j}^{(l)}$ to the vector $\bar{s}_{i-1}$, and that this $\bar{s}_{i-1}$ is known to the predictor at the time of computing its prediction for $\bar{s}_{i}$. Thus, each of the sequences $\left\{s_{i,}\right\}_{i=0}^{\infty}, l=1, \ldots, n$ are efficiently predictable and so is the whole vector sequence. The number of possible prediction errors is as the sum of possible errors in each of the sub-generators $\Phi^{(l)}$. That is, at most $n$ times the bound of Theorem 4 .

One can take advantage of the fact that the different sub-generators work with the same modulus $m$ in order to accelerate the convergence to the true value of $m$. At the end of each prediction step, we have $n$ (not necessarily different) estimates $\hat{m}^{(1)}, \ldots, \hat{m}^{(n)}$ 
computed by the predictors for $\Phi^{(1)}, \ldots, \Phi^{(n)}$, respectively. In the next prediction we put all the predictors to work with the same estimate $\hat{m}$ computed as $\hat{m}=\operatorname{gcd}\left(\hat{m}^{(1)}, \ldots, \hat{m}^{(n)}\right)$ . This works since each of the $m^{(l)}$ is guaranteed to be a multiple of $m$ (claim (a) in Lemmas 1 and 3). In this way we get that the total number of mistakes is bounded by $(n k+1)\left(\log m_{0}+1\right)$. Notice that the dimension of the whole system of equations corresponding to the $n \Phi^{(l)}$-generators is $n k$ (as is the total number of coefficients hidden from the predictor). On the other hand, the bound on $\hat{m}_{0}$ from Lemma 1 is still valid. It does not depend on the number of sub-generators since we predict each $\Phi^{(l)}$-generator (i.e. solve the corresponding system of equations) separately. Thus, we can restate Theorem 4 for the vector-valued case.

Theorem 5: Vector-valued recurrences of the form (4) are efficiently predictable provided that each $\Phi^{(l)}$-generator, $l=1, \ldots, n$, has polynomial-time non-reduced complexity. The number of mistakes made by the above predicting algorithm is $O\left(n k^{2} \log (k m M)\right)$, where $M$ is an upper bound on the output of each of the functions $\Phi_{j}^{(l)}, j=1, \ldots, k, l=1, \ldots, n$, working over integers in the set $\{0, \ldots, m-1\}$. In particular, for vector-valued polynomial recurrences in $n$ variables and degree at most $d$ the number of mistakes is $O\left(n k^{2} \log \left(k m^{d}\right)\right)$, where $k=\left(\begin{array}{c}n+d \\ d\end{array}\right)$.

Remark: For simplicity we have restricted ourselves to the case (4) in which the subgenerators $\phi^{(l)}$ work on the last vector $\bar{s}_{i-1}$. Clearly, our results hold for the more general case in which each of these sub-generators may depend on the whole vector sequence $\bar{s}_{-n_{0}}, \ldots, \bar{s}_{i-1}$ output so far. In this case the number $n$ of sub-generators does not depend on the number of arguments the sub-generators work on, and the number of arguments does not effect the number of mistakes.

\section{CONCLUDING REMARKS}

Our prediction results concern number generators outputting all the bits of the generated numbers, and does not apply to generators that output only parts of the numbers generated. Recent works treat the problem of predicting linear congruential generators which output only parts of the numbers generated $[9,14,19]$.

A theorem by Yao [21] states that pseudorandom (bit) generators are unpredictable by polynomial-time means if and only if they pass any polynomial time statistical test. That is, predictability is a universal statistical test in the sense that if a generator is unpredictable, then it will pass any statistical test. Thus, a generator passing this universal test will be suitable for any "polynomially bounded" application. Nevertheless, for specific applications, some weaker generators may suffice. As an example, for their use in some simulation processes, all that is required from the generators is some distribution properties of the numbers generated. In the field of Probabilistic Algorithms the correctness of the algorithm is often analyzed assuming the total randomness of the coin tosses 
of the algorithm. However, in special cases a more relaxed assumption is possible. For example Bach [2] shows that simple linear congruential generators suffice for guaranteeing the correctness and efficiency of some probabilistic algorithms, even though these generators are clearly predictable. In [7] linear congruential generators are used to "expand randomness". Their method allows the deterministic "expansion" of a truly random string into a sequence of pairwise independent pseudorandom strings.

Provable unpredictable generators exist, assuming the existence of one-way functions $[4,21,10,11]$. In particular, assuming the intractability of factoring, the following pseudorandom bit generator is unpredictable $[5,1,20]$. This generator outputs a bit sequence $b_{1}, b_{2}, \ldots$, where $b_{i}$ is the least significant bit of $s_{i}, s_{i} \equiv s_{i-1}^{2}(\bmod m)$, and $m$ is the product of two large primes.

\section{ACKNOWLEDGEMENTS}

I wish to thank Oded Goldreich for his help and guidance during the writing of this paper, and for many other things I have learned from him. Also, I would like to thank Johan Hastad for suggesting an improvement to my original bound on the number of prediction mistakes.

\section{REFERENCES}

[1] Alexi, W., B. Chor, O. Goldreich and C.P. Schnorr, RSA and Rabin Functions: Certain Parts Are As Hard As the Whole, SIAM J. Comput., Vol. 17, 1988, pp. 194-209.

[2] Bach, E., Realistic Analysis of Some Randomized Algorithms, Proc. 19th ACM Symp. on Theory of Computing, 1987, pp. 453-461.

[3] Boyar, J. Inferring Sequences Produced by Pseudo-Random Number Generators, Jour. of $A C M$, Vol. 36, No. 1, 1989, pp.129-141.

[4] Blum, M., and Micali, S., How to Generate Cryptographically Strong Sequences of Pseudo-Random Bits, SIAM J. Comput., Vol. 13, 1984, pp. 850-864.

[5] Blum, L., Blum, M., and Shub, M., A Simple Unpredictable Pseudo-Random Number Generator, SIAM J. Comput., Vol. 15, 1986, pp. 364-383.

[6] Butson, A.T., and Stewart, B.M., Systems of Linear Congruences, Canad. J. Math., Vol. 7, 1955, pp. 358-368.

[7] Chor, B., and Goldreich, O., On the Power of Two-Points Based Sampling, Jour. of Complexity, Vol. 5, 1989, pp. 96-106.

[8] Edmonds, J., Systems of Distinct Representatives and Linear Algebra, Journal of Research of the National Bureau of Standards (B), Vol. 71B, 1967, pp. 241-245. 
[9] Frieze, A.M., Hastad, J., Kannan, R., Lagarias, J.C., and Shamir, A. Reconstructing Truncated Integer Variables Satisfying Linear Congruences SIAM J. Comput., Vol. 17, 1988, pp. 262-280.

[10] Goldreich, O., H. Krawczyk and M. Luby, "On the Existence of Pseudorandom Generators", Proc. 29th IEEE Symp. on Foundations of Computer Science, 1988, pp 12-24.

[11] Impagliazzo, R., L.A., Levin and M.G. Luby, "Pseudo-Random Generation from OneWay Functions", Proc. 21 th ACM Symp. on Theory of Computing, 1989, pp. 12-24.

[12] Kannan, R., and Bachem, A., Polynomial Algorithms for Computing the Smith and Hermite Normal Forms of an Integer Matrix, SIAM J. Comput., Vol. 8, 1979, pp. 499-507.

[13] Knuth, D.E., "The Art of Computer Programming, Vol. 2: Seminumerical Algorithms", Addison-Wesley, Reading, Mass., 1969.

[14] Knuth, D.E., Deciphering a Linear Congruential Encryption, IEEE Trans. Info. Th. IT31, 1985, pp. 49-52.

[15] Lagarias, J.C., and Reeds, J., Unique Extrapolation of Polynomial Recurrences, SIAM J. Comput., Vol. 17, 1988, pp. 342-362.

[16] Plumstead (Boyar), J.B., Inferring a Sequence Generated by a Linear Congruence, Proc. of the 23rd IEEE Symp. on Foundations of Computer Science, 1982, pp. 153-159.

[17] Plumstead (Boyar), J.B., Inferring Sequences Produced by Pseudo-Random Number Generators, Ph.D. Thesis, University of Califomia, Berkeley, 1983.

[18] Schrijver, A., "Theory of Linear and Integer Programming". Willey, Chichester, 1986.

[19] Stern, J., Secret Linear Congruential Generators Are Not Cryptographically Secure, Proc. of the 28rd IEEE Symp. on Foundations of Computer Science, 1987.

[20] Vazirani, U.V., and Vazirani, V.V., Efficient and Secure Pseudo-Random Number Generation, Proc. of the 25th IEEE Symp. on Foundations of Computer Science, 1984, pp. 458-463.

[21] Yao, A.C., Theory and Applications of Trapdoor Functions, Proc. of the 23rd IEEE Symp. on Foundations of Computer Science, 1982, pp. 80-91. 